\title{
Maternal LC-PUFA status during pregnancy and child problem behavior: the Generation R Study
}

\author{
Jolien C.J. Steenweg-de Graaff ${ }^{1,2}$, Henning Tiemeier ${ }^{2-4}$, Maartje G.J. Basten ${ }^{1,2}$, Jolien Rijlaarsdam ${ }^{1,2}$, Hans Demmelmair ${ }^{5}$,
} Berthold Koletzko ${ }^{5}$, Albert Hofman ${ }^{3}$, Vincent W.V. Jaddoe ${ }^{1,3,6}$, Frank C. Verhulst ${ }^{2}$ and Sabine J. Roza ${ }^{2,4}$

BACKGROUND: Omega 3 (n-3) and 6 (n-6) long-chain polyunsaturated fatty acids (LC-PUFAs) and the $n-3: n-6$ ratio are important for brain development. Whether maternal LC-PUFA status during pregnancy affects risk of problem behavior in later childhood is unclear.

METHODS: Within a population-based cohort, we measured maternal plasma docosahexaenoic acid (DHA), eicosapentaenoic acid (EPA), and arachidonic acid (AA) concentrations and n-3:n-6-ratio in mid-pregnancy. Child emotional and behavioral problems at 6 y of age were assessed by parents (child behavior checklist), teachers (teacher report form), and combined parent/teacher report.

RESULTS: Higher maternal DHA and n-3:n-6 ratio were associated with fewer child emotional problems using parent (odds ratio $(\mathrm{OR})_{\mathrm{DHA}}=0.82 ; 95 \%$ confidence interval $(\mathrm{Cl}): 0.70,0.96$; $P=0.02$ and $\mathrm{OR}_{n-3.3-6}=0.83 ; 95 \% \mathrm{Cl}: 0.71,0.96 ; P=0.01 ; n=$ $5,307)$ and combined parent/teacher scores $\left(\mathrm{OR}_{\mathrm{DHA}}=0.79 ; 95 \%\right.$ $\mathrm{Cl}: 0.66,0.95 ; P=0.01$ and $\mathrm{OR}_{n-3 n-6}=0.77 ; 95 \% \mathrm{Cl}: 0.65,0.92 ; P$ $<0.01 ; n=2,828)$. Higher AA was associated with more child behavioral problems using teacher $(\mathrm{OR}=1.10 ; 95 \% \mathrm{Cl}$ : 1.00 , 1.20; $P=0.04 ; n=3,365$ ) and combined parent/teacher scores $(\mathrm{OR}=1.12 ; 95 \% \mathrm{Cl}: 1.02,1.22 ; P=0.02 ; n=2,827)$. Maternal EPA was not associated with child problem behavior.

CONCLUSION: Indications of associations of maternal LC-PUFA status with child emotional and behavioral problems were found. Future research is needed to identify LC-PUFAsensitive periods of fetal brain development by including multiple assessments of prenatal LC-PUFA status.

$T$ wo families of polyunsaturated fatty acids (PUFAs), the omega $3(n-3)$ and $6(n-6)$ fatty acids (FAs), are nutrients that play a major role in brain development and functioning. They affect numerous processes, including membrane fluidity, neurotransmission, and gene expression (1). The long-chain PUFAs (LC-PUFAs), eicosapentaenoic acid (EPA; C20:5n-3), docosahexaenoic acid (DHA; C22:6n-3), and arachidonic acid (AA; C20:4n-6) are the main FAs that are metabolically active.
In addition, the ratio between $n-3$ and n-6 FAs is important for optimal brain functioning. During the last century, however, the Western diet has provided a lower n-3 and higher $\mathrm{n}-6$ intake than that in previous generations, diminishing the n-3:n-6 ratio (2).

Because of their role in brain function and development, maternal LC-PUFAs during fetal development have been hypothesized to affect child problem behavior. Approximately half of the dry weight of the brain is lipid, of which $35 \%$ are PUFA, most of which is LC-PUFA (1). Increase in AA and DHA content of brain tissue starts during the third trimester of pregnancy (3), indicating that the fetal period possibly is a sensitive period. Moreover, the fetus mostly depends on maternal LC-PUFA supply for its development, because fetal LC-PUFA synthesis is limited (4).

Research in this field is however scarce, and results are conflicting. Two studies on maternal fish intake, a major source of DHA, during pregnancy and offspring problem behavior were undertaken. They found low maternal fish intake to be associated with suboptimal prosocial behavior and increased risk of hyperactivity in childhood $(5,6)$. Another study on dietary intake of n-3 LC-PUFAs did not find any association with child behavior (7). Animal studies have shown that prenatal and not postnatal DHA deficiency was associated with increased depression and anxiety later in life (8). In humans, there is some indication that perinatal LC-PUFA status (either measured in cord blood or maternal blood during gestation) is associated with child behavior. However, results are inconsistent, ranging from null findings to associations with both emotional and behavioral problems (9-12). Most studies were small and probably underpowered to detect subtle effects of nutrition, as expected in a multicausal phenomenon such as development of the brain and behavior. Moreover, multiple informants-the gold standard in child psychiatric research to measure a child's core characteristics from different perspectives and in different contexts-were generally not used.

A recent, relatively large study in another birth cohort from the Netherlands that did use multiple informants

\footnotetext{
'The Generation R Study Group, Erasmus Medical Center, Rotterdam, The Netherlands; ${ }^{2}$ Department of Child and Adolescent Psychiatry/Psychology, Erasmus MC-Sophia, Rotterdam, The Netherlands; ${ }^{3}$ Department of Epidemiology, Erasmus Medical Center, Rotterdam, The Netherlands; ${ }^{4}$ Department of Psychiatry, Erasmus Medical Center, Rotterdam, The Netherlands; ${ }^{5}$ Division of Metabolic and Nutritional Medicine, Dr. von Hauner Children's Hospital, University of Munich Medical Center, Munich, Germany; ${ }^{6}$ Department of Pediatrics, Erasmus MC-Sophia, Rotterdam, The Netherlands. Correspondence: Sabine J. Roza (s.roza@erasmusmc.nl) 


\section{Articles | Steenweg-de Graaff et al.}

reported a modest association between maternal DHA level and child emotional symptoms (12). In a study twice as large, with extended behavioral assessment by multiple informants and the availability of Diagnostic and Statistical Manual of Mental Disorders (DSM-IV) diagnoses of problem behavior, we aimed to further study the effects of maternal LC-PUFAs during pregnancy on child problem behavior. To this end, we tested the hypotheses that maternal LC-PUFA status is associated with child emotional and behavioral problems at $6 \mathrm{y}$ of age. Because of their metabolic role in brain development, we focused on AA, EPA, and DHA, as well as the n-3:n-6 ratio.
RESULTS

Descriptive characteristics of study participants by determinant and outcome are presented in Tables 1 and 2 respectively.

\section{Maternal LC-PUFAs and Child Emotional Problems}

The fully adjusted associations between maternal LC-PUFA status and child emotional problems, as reported by different informants and assessed with different instruments, are presented in Table 3. No associations of maternal AA or EPA with child emotional problems were found. Increased levels of maternal DHA were associated with fewer child emotional problems as reported by parents (child behavior checklist

Table 1. Subject characteristics by maternal n-3:n-6 ratio in mid-pregnancy ${ }^{b}$

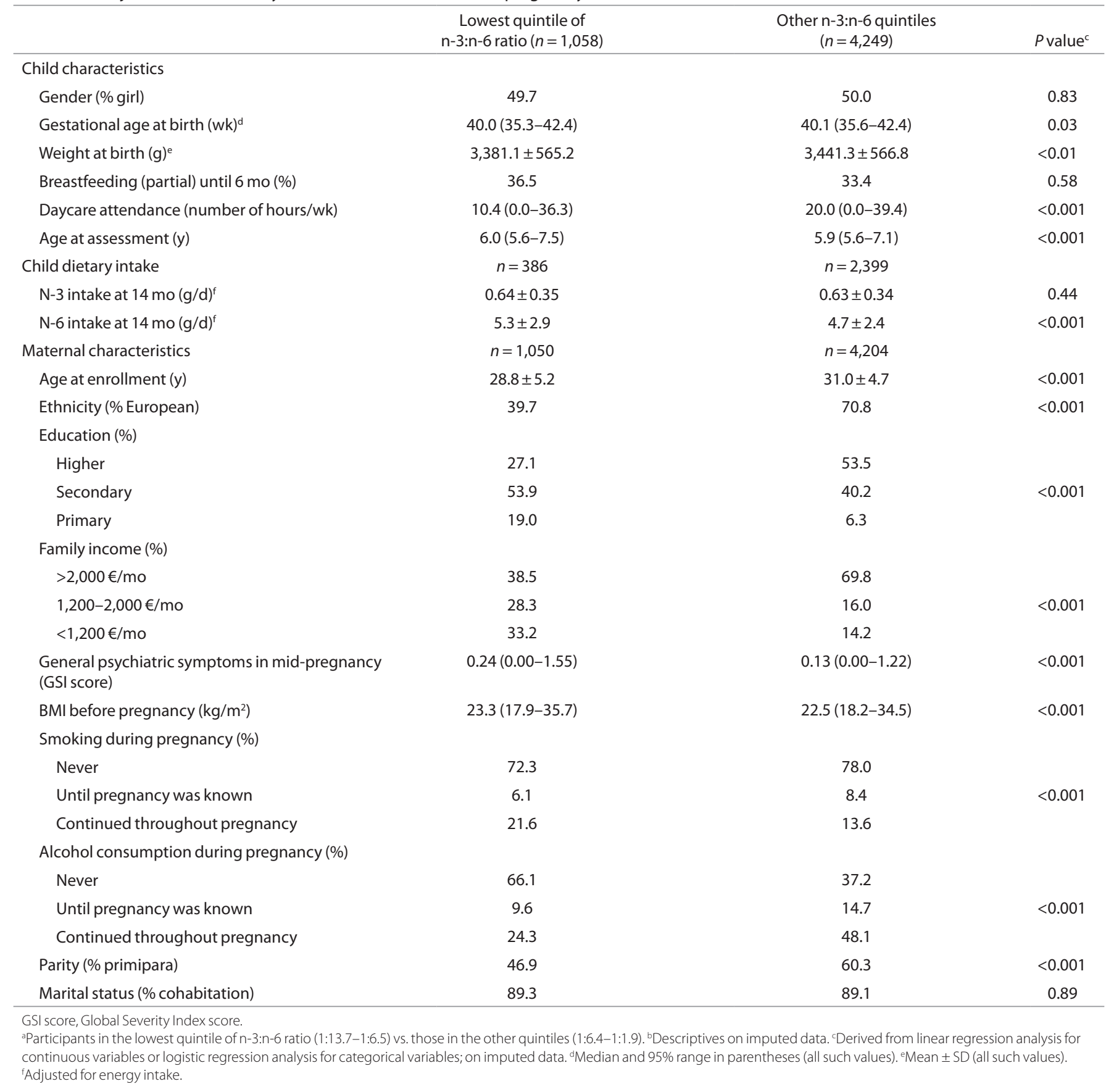


$(\mathrm{CBCL})$; odds ratio $(\mathrm{OR})_{\text {parent score }}=0.82 ; 95 \%$ confidence inter$\operatorname{val}(\mathrm{CI}): 0.70,0.96 ; P=0.02)$ and using the combined scores of both parents (CBCL) and teachers (teacher report form (TRF); $\left.\mathrm{OR}_{\text {combined score }}=0.79 ; 95 \% \mathrm{CI}: 0.66,0.95 ; P=0.01\right)$. Similarly, a higher n-3:n-6 ratio was associated with a lower risk of emotional problems $\left(\mathrm{OR}_{\text {parent score }}=0.83 ; 95 \% \mathrm{CI}\right.$ : 0.71, 0.96; $P=0.01$ and $\left.\mathrm{OR}_{\text {combined score }}=0.77 ; 95 \% \mathrm{CI}: 0.65,0.92 ; P<0.01\right)$. In contrast, no associations of maternal DHA or $n-3: n-6$ ratio with child emotional problems based on teacher report only were found $\left(\mathrm{OR}_{\mathrm{DHA}}=1.04 ; 95 \% \mathrm{CI}: 0.86,1.25 ; \mathrm{P}=0.70\right.$ and $\mathrm{OR}_{\mathrm{n}-3: \mathrm{n}-6}$ $=0.98 ; 95 \%$ CI: $0.82,1.17 ; P=0.84)$. Also, no associations of maternal fatty acids with offspring emotional problems were found using the parent-administered Diagnostic Interview Schedule for Children-Young Child version (DISC-YC; OR $=0.82 ; 95 \%$ CI: $0.57,1.18 ; P=0.28$ and $\mathrm{OR}_{\mathrm{n}-3: \mathrm{n}-6}=0.94 ; 95 \% \mathrm{CI}$ : $0.65,1.38 ; P=0.76)$.

Including quadratic terms of the FAs in the model did not suggest nonlinear associations, and results did not change

Table 2. Subject characteristics by child total emotional and behavioral problems $s^{\mathrm{a}}$ at $6 \mathrm{y}^{\mathrm{b}}$

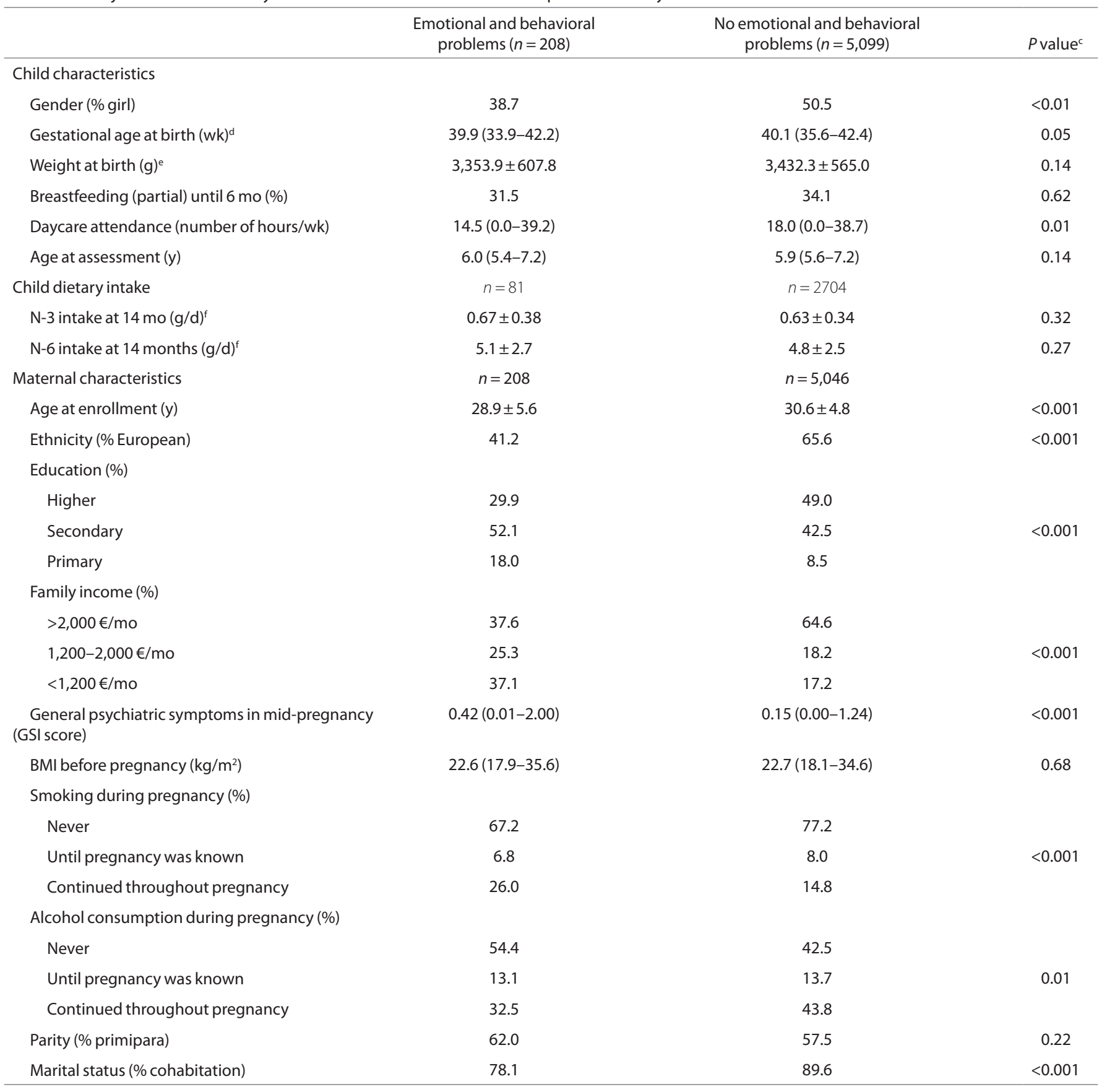


Table 3. Associations of maternal prenatal fatty acids with child emotional and behavioral problems at $6 y^{a}$

\begin{tabular}{|c|c|c|c|c|c|c|c|c|}
\hline & \multicolumn{8}{|c|}{ Emotional problems } \\
\hline & \multicolumn{2}{|c|}{$\begin{array}{l}\text { Parent report } \\
(n=5,307)^{\mathrm{b}}\end{array}$} & \multicolumn{2}{|c|}{$\begin{array}{l}\text { Teacher report } \\
\quad(n=3,366)^{c}\end{array}$} & \multicolumn{2}{|c|}{$\begin{array}{l}\text { Combined parent and teacher } \\
\text { report }(n=2,828)^{d}\end{array}$} & \multicolumn{2}{|c|}{$\begin{array}{l}\text { Parent interview, DSM- } \\
\text { IV-based diagnosis } \\
(n=490)^{\mathrm{e}}\end{array}$} \\
\hline & $\mathrm{OR}^{\mathrm{g}}(95 \% \mathrm{Cl})$ & $P$ & $\mathrm{OR}^{\mathrm{g}}(95 \% \mathrm{Cl})$ & $P$ & $\mathrm{OR}^{\mathrm{g}}(95 \% \mathrm{Cl})$ & $P$ & $\mathrm{OR}^{\mathrm{g}}(95 \% \mathrm{Cl})$ & $P$ \\
\hline \multicolumn{9}{|l|}{ Maternal fatty acid $(w t \%)^{f}$} \\
\hline EPA & $1.09(0.65,1.86)$ & 0.74 & $0.60(0.28,1.28)$ & 0.19 & $1.00(0.53,1.89)$ & 0.99 & $1.83(0.64,5.23)$ & 0.26 \\
\hline DHA & $0.82(0.70 ; 0.96)$ & 0.02 & $1.04(0.86,1.25)$ & 0.70 & $0.79(0.66,0.95)$ & 0.01 & $0.82(0.57,1.18)$ & 0.28 \\
\hline \multirow[t]{2}{*}{ N-3:N-6 ratio (per SD) } & $0.83(0.71,0.96)$ & 0.01 & $0.98(0.82,1.17)$ & 0.84 & $0.77(0.65,0.92)$ & $<0.01$ & $0.94(0.65,1.38)$ & 0.76 \\
\hline & \multicolumn{8}{|c|}{ Behavioral problems } \\
\hline \multicolumn{9}{|l|}{ Maternal fatty acid $(w t \%)^{f}$} \\
\hline $\mathrm{AA}$ & $1.04(0.91,1.18)$ & 0.60 & $1.10(1.00,1.20)$ & 0.04 & $1.12(1.02,1.22)$ & 0.02 & $1.07(0.95,1.22)$ & 0.25 \\
\hline EPA & $1.58(0.84,2.95)$ & 0.15 & $0.85(0.45,1.58)$ & 0.60 & $1.22(0.70,2.10)$ & 0.48 & $1.09(0.59,2.02)$ & 0.79 \\
\hline DHA & $0.93(0.75,1.16)$ & 0.53 & $1.23(1.03,1.46)$ & 0.02 & $1.07(0.90,1.26)$ & 0.47 & $0.89(0.72,1.11)$ & 0.30 \\
\hline N-3:N-6 ratio (per SD) & $1.09(0.88,1.34)$ & 0.44 & $1.23(1.05,1.44)$ & 0.01 & $1.16(0.99,1.36)$ & 0.06 & $0.89(0.73,1.08)$ & 0.24 \\
\hline \multicolumn{9}{|c|}{ 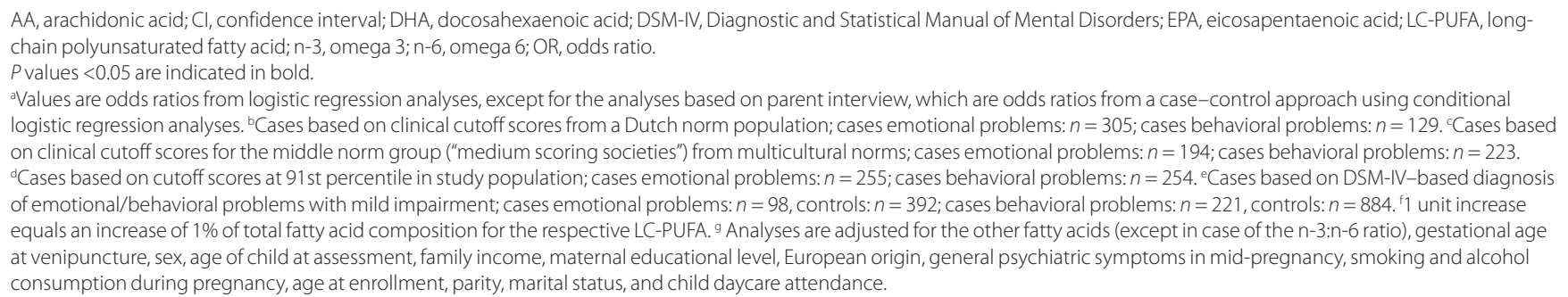 } \\
\hline
\end{tabular}

after exclusion of preterm children and children with low birth weight.

The analyses were repeated while adjusting for dietary n-3 and n-6 intake at 14 mo of age in a subsample with these data available $\left(\mathrm{n}_{\text {parent score }}=2,785\right)$. Dietary $n-3$ and $n-6$ data did not change the effect size of DHA nor the n-3:n-6 ratio on child emotional problems (in this subsample, before and after adjustment for child diet: $\mathrm{OR}_{\mathrm{DHA}}=0.75 ; 95 \%$ CI: $0.59,0.96 ; P=$ 0.02 and $\mathrm{OR}_{\mathrm{n}-3 \mathrm{n}-\mathrm{n}}=0.74 ; 95 \%$ CI: $0.59,0.93 ; P=0.01$ ).

With regard to potential effect modification, the interaction term of maternal n-3:n-6 ratio with child sex, but not maternal educational level, was statistically significant $(P<0.05)$. Analyses stratified by sex showed that the effect of maternal n-3:n-6 ratio on child emotional problems could be attributed to an effect in girls $\left(\mathrm{OR}_{\text {girls }}=0.71 ; 95 \% \mathrm{CI}: 0.56,0.91 ; P<0.01\right.$ and $\mathrm{OR}_{\text {boys }}=0.91 ; 95 \%$ CI: $0.74,1.12 ; P=0.36$ ).

In Table 4, we show the association of maternal n-3:n-6 ratio divided by quintiles with child emotional problems. The lowest quintile was used as the reference category (quintile 1). Compared with this reference, a maternal n-3:n-6 ratio in the highest two quintiles was associated with fewer emotional problems in the offspring $\left(\mathrm{OR}_{\mathrm{Q} 4 \mathrm{vs} 1}=0.62 ; 95 \% \mathrm{CI}: 0.40,0.95\right.$; $P=0.03$ and $\mathrm{OR}_{\mathrm{Q} 5 v \mathrm{vQ} 1}=0.61$; 95\% CI: $0.39,0.96 ; P=0.03$ ).

\section{Maternal LC-PUFAs and Child Behavioral Problems}

The fully adjusted associations between maternal LC-PUFA status and child behavioral problems are presented in Table 3. No associations were found between maternal EPA and child behavioral problems. Higher maternal DHA level and n-3:n-6 ratio were only associated with more child behavioral problems using the teacher report $\left(\mathrm{OR}_{\mathrm{DHA}}=1.23\right.$; 95\% CI: $1.03,1.46 ; P=0.02 ; \mathrm{OR}_{\mathrm{n}-3: \mathrm{n}-6}=1.23 ; 95 \%$ CI: 1.05 , $1.44 ; P=0.01)$.

However, for both the teacher score and the combined parent/teacher score, increased levels of maternal AA were associated with more child behavioral problems $\left(\mathrm{OR}_{\text {teacher score }}=1.10\right.$; 95\% CI: $1.00,1.20 ; P=0.04$ and $\mathrm{OR}_{\text {combined score }}=1.12 ; 95 \% \mathrm{CI}$ : $1.02,1.22 ; P=0.02)$. These positive associations were not confirmed in analyses using parent report only (either CBCL or DISC-YC interview).

Again, including quadratic terms of the FAs in the model did not suggest nonlinear associations, and results did not change 
Table 4. Associations of maternal prenatal $n-3: n-6$ ratio with child emotional and behavioral problems at $6 \mathrm{y}^{\mathrm{a}}$

\begin{tabular}{|c|c|c|c|c|}
\hline \multirow[b]{3}{*}{$\begin{array}{l}\text { Maternal n-3:n-6 } \\
\text { ratio }\end{array}$} & \multicolumn{4}{|c|}{ Emotional problems $(n=5,307)^{\mathrm{b}}$} \\
\hline & \multicolumn{2}{|c|}{ Basic $^{c}$} & \multicolumn{2}{|c|}{$\begin{array}{l}\text { Adjusted for } \\
\text { covariates }^{\mathrm{d}}\end{array}$} \\
\hline & OR $(95 \% \mathrm{Cl})$ & $P$ & OR $(95 \% \mathrm{Cl})$ & $P$ \\
\hline \multicolumn{5}{|l|}{ Quintiles } \\
\hline $1(1: 13.7-1: 6.5)$ & Reference & & Reference & \\
\hline $2(1: 6.4-1: 5.6)$ & $0.81(0.58,1.13)$ & 0.22 & $1.02(0.72,1.44)$ & 0.91 \\
\hline $3(1: 5.5-1: 4.9)$ & $0.58(0.40,0.86)$ & $<0.01$ & $0.82(0.55,1.22)$ & 0.33 \\
\hline $4(1: 4.8-1: 4.2)$ & $0.39(0.25,0.59)$ & $<0.001$ & $0.62(0.40,0.95)$ & 0.03 \\
\hline $5(1: 4.1-1: 1.9)$ & $0.35(0.23,0.53)$ & $<0.001$ & $0.61(0.39,0.96)$ & 0.03 \\
\hline \multirow{2}{*}{$\begin{array}{l}\text { Maternal n-3:n-6 } \\
\text { ratio }\end{array}$} & \multicolumn{4}{|c|}{ Behavioral problems $(n=5,307)^{\mathrm{b}}$} \\
\hline & OR $(95 \% \mathrm{Cl})$ & $P$ & OR $(95 \% \mathrm{Cl})$ & $P$ \\
\hline \multicolumn{5}{|l|}{ Quintiles } \\
\hline $1(1: 13.7-1: 6.5)$ & Reference & & Reference & \\
\hline $2(1: 6.4-1: 5.6)$ & $1.24(0.64,2.42)$ & 0.52 & $1.35(0.70,2.60)$ & 0.37 \\
\hline $3(1: 5.5-1: 4.9)$ & $1.12(0.60,2.10)$ & 0.71 & $1.31(0.70,2.43)$ & 0.40 \\
\hline $4(1: 4.8-1: 4.2)$ & $0.77(0.39,1.53)$ & 0.45 & $1.02(0.51,2.06)$ & 0.95 \\
\hline $5(1: 4.1-1: 1.9)$ & $0.86(0.45,1.65)$ & 0.66 & $1.28(0.65,2.54)$ & 0.48 \\
\hline \multicolumn{5}{|c|}{ 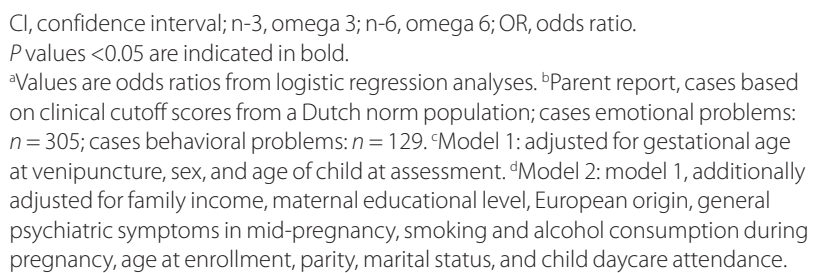 } \\
\hline
\end{tabular}

after exclusion of preterm children and children with low birth weight.

Adjustment for child n-3 and n-6 intake in a subsample $\left(\mathrm{n}_{\text {teacher score }}=1,582\right.$ and $\left.\mathrm{n}_{\text {combined score }}=1,553\right)$ did not change the association of AA with child behavioral problems using the teacher report (in this subsample, before and after adjustment for child diet: $\mathrm{OR}_{\mathrm{AA}}=1.22 ; 95 \% \mathrm{CI}: 1.04,1.43 ; P=0.02$ ). The association between AA and child behavior using the combined parent/teacher report in this subsample did not reach level of statistical significance, either before or after adjustment for child dietary intake $\left(\mathrm{OR}_{\mathrm{AA}}=1.13 ; 95 \% \mathrm{CI}: 0.99,1.30 ; P=0.07\right)$.

\section{DISCUSSION}

In this population-based study, we found higher maternal DHA levels and n-3:n-6 ratio to be associated with fewer offspring emotional problems using both a parent and combined parent/teacher report of child problem behavior. This association could not be confirmed using teacher report only or using the DISC-YC interview. If anything, we interpret these findings as an indication of a negative association of maternal DHA levels and n-3:n-6 ratio with offspring emotional problems.

Similarly, we found higher maternal AA levels to be associated with more child behavioral problems using both teacher and combined parent/teacher report. The associations using parent report and DISC-YC data were statistically null findings. These findings indicate a positive association of maternal AA levels with offspring behavioral problems.

No associations between maternal EPA levels and child emotional or behavioral problems were found.

\section{Interpretation}

Our findings closely resemble those from Loomans et al. (12), who also found higher maternal DHA-but not AA or EPAto be associated with fewer child emotional symptoms at the age of 5-6 y based on combined report of mother and teacher using the Strength and Difficulties Questionnaire. We additionally found indications of higher maternal n-3:n-6 ratio and AA levels to be associated with, respectively, fewer emotional and more behavioral problems in the offspring, whereas these associations disappeared after adjustment for confounders in the study by Loomans et al. Our study, however, has more power. Additionally, our measures of behavioral problems also comprise aggressive and rule-breaking behaviors, whereas the Strength and Difficulties Questionnaire only measures hyperactivity and inattention.

Our results are also compatible with findings from Krabbendam et al. (10), who found higher DHA but not AA cord blood levels to be associated with fewer emotional problems in children at the age of $7 \mathrm{y}$ based on CBCL parent report. Moreover, like Krabbendam et al., who found no association between current child LC-PUFA status and child problem behavior, we did not find infant n-3 and n-6 intake to affect the associations between maternal LC-PUFAs and child emotional or behavioral problems at the age of $6 \mathrm{y}$. This stresses the potential independent effect of prenatal LC-PUFA status on child emotional and behavioral development.

Our results are generally not in line with those from Kohlboeck et al. (11), who found higher levels of AA in cord blood to be associated with fewer emotional problems at the age of $10 \mathrm{y}$ based on parent report using the Strength and Difficulties Questionnaire. Whether statistically significant or not, all associations of maternal AA with child problem behavior in our study suggest effect estimates in the opposite direction, as do results from other studies $(9,10,12)$. Kohlboeck et al. also found higher levels of DHA to be associated with less hyperactivity/inattention, whereas we found an indication of higher maternal AA levels to be associated with more behavioral problems. Differences in findings may be attributable to a combination of differences in fatty acid assessment (cord blood serum vs. mid-pregnancy plasma sample), instrument and informant of child problem behavior (parent Strength and Difficulties Questionnaire vs. multiinformant assessment by CBCL, TRF, and DISC-YC interview), and child age at assessment (10 vs. 6 y of age).

Finally, our results are not consistent with those from Dunstan et al. (9), who reported no significant associations between cord blood LC-PUFAs and problem behavior at the age of $2.5 \mathrm{y}$ using CBCL parent report. Our study, however, has a much larger sample size and thus increased power to detect associations. Additionally, our study has a longer follow-up 


\section{Articles | Steenweg-de Graaff et al.}

time, during which parents may become better in recognizing and thus reporting problem behavior of their child and during which other informants of child problem behavior, such as teachers, become available.

In contrast to the studies mentioned above, we provide an overview of the associations of maternal LC-PUFAs with child problem behavior as reported by different informants. There is a large body of literature indicating the important variability in behavior across different contexts, such as home vs. school (13). Our approach increases insight in the effect that the difference in multi-informant agreement on children's problem behavior may have on associations under investigation. As a consequence, however, this approach makes it harder to make strong statements when results do not all point toward same conclusion. Therefore, not only findings from studies using one informant/instrument but also findings from the current study should be interpreted with caution.

\section{Mechanism of Risk}

Animal studies have shown that maternal dietary LC-PUFA composition may durably alter brain monoamine, dopamine, and serotonin concentrations in the offspring $(14,15)$. Moreover, such modifications in 2-mo-old rats were only reversed by supply of a LC-PUFA-balanced diet that started before weaning (15), when rat brain development is similar to that of a full-term human newborn (16). It is known in humans that maternal LC-PUFA status declines during pregnancy (17), and LC-PUFAs accumulate in the fetal brain, with a spurt in the last trimester of pregnancy (1). However, maternal LC-PUFA status may also affect brain development earlier in pregnancy. Gale et al. (6) found oily fish intake during early but not late pregnancy to be associated with less hyperactivity in children. Lower maternal LC-PUFA concentrations directly limit the amount of LC-PUFA available to the fetus (4), and animal studies showed that already early in pregnancy LC-PUFAs promote neurogenesis and then continue to influence synaptogenesis and myelination (18-20). The developmental stage in which inadequate LC-PUFA status occurs during pregnancy may thus additionally account for different effects of LC-PUFA status.

Alternatively, maternal depression underlies the observed association of maternal LC-PUFAs with child problem behavior. Antenatal depression is an established cause of child emotional and behavioral problems (21). Low LC-PUFA status might place women at risk of depression or can result from dietary changes due to depression (22). However, we adjusted for maternal depressive symptoms during pregnancy to control for such an indirect effect.

Finally, this type of nutritional associations in observational studies is sensitive to effects of confounding and effect-modifying factors. A decline in the effect of maternal LC-PUFA status on the offspring's problem behavior was noticeable after adjustment for confounders. Admitting we were not able to adjust for child LC-PUFA concentrations, we found no evidence that child dietary intake of n-3 and $n-6$ FAs influenced the associations. Effect-modification by sex further points to a complex causal chain, in which many factors exaggerate effects on child emotional and behavioral development. Although we accounted for many sociodemographic factors, further (residual) confounding cannot be excluded.

\section{Strengths and Limitations}

The strengths of our study were its large sample size, the availability of plasma LC-PUFA concentrations, the multi-informant assessment of child behavior, and the ability to adjust for several potential confounders.

The study also has its limitations. Selective attrition may have influenced our results. Mothers of children not included generally had less favorable LC-PUFA status and socioeconomic circumstances. This selective attrition can only lead to bias if associations of maternal fatty acids and child emotional/ behavioral problems in those mother-child pairs not included in the study differ from the associations found in those that were included in the study. With regard to the hypothesized biological mechanism underlying the association, we do not consider this likely. It can however not be inferred from our results. Next, our assessment of LC-PUFA status was based on a single measure in blood plasma in mid-pregnancy. One measurement, although indicative, is not a reliable reflection of a mother's long-term FA status. Furthermore, other nutrients that are associated with brain development and that simultaneously affect FA status, such as iron, were not included in this study (18).

\section{Conclusion}

We found indications of higher maternal DHA levels and n-3:n-6 ratio during second trimester of pregnancy to be associated with fewer emotional problems and higher AA levels with more behavioral problems in the offspring at the age of $6 \mathrm{y}$. These findings are a step forward for the growing body of literature in this field and underline the importance of the use of multiple informants of child problem behavior in association studies like these. Further research is needed to identify LC-PUFA-sensitive periods of fetal brain development, optimal intake of LC-PUFAs during pregnancy, and the specific effects of inadequate prenatal LC-PUFA status on emotional and behavioral development in the offspring. These studies should include multiple assessments of FAs during pregnancy, as well as in-depth assessments of child problem behavior by multiple informants and instruments.

\section{METHODS}

\section{Study Population}

The present study was conducted within the Generation R Study, a population-based cohort from early fetal life onwards in Rotterdam, The Netherlands (23). The study was conducted in accordance with the guidelines proposed in the World Medical Association Declaration of Helsinki and was approved by the Medical Ethical Committee of the Erasmus Medical Center. Written consent was obtained from all participants.

Out of 8,663 mothers who enrolled in the study before their third trimester of pregnancy $(<25 \mathrm{wk}$ of gestation), valid FA profiles in plasma were available for 6,999 mothers (80.8\%). Overall, 6,916 
mother-child pairs were eligible at study baseline. In total, at least one measure of child behavior was available for 5,307 children (76.7\%) and their mothers (Figure 1). Some mothers participated with two $(n=309)$ or three $(n=6)$ children. Since results of all analyses using parent report did not differ after exclusion of twins $(n=110)$ or random exclusion of one or two of the siblings, they were kept in the analyses.

\section{Fatty Acid Analyses}

Between 2001 and 2005, venous samples were drawn in mid-pregnancy (median: $20.5 \mathrm{wk}$ of gestation; 90\% range: $18.8-22.9 \mathrm{wk}$ ) and thereafter stored at room temperature before being transported to the regional laboratory for processing and storage for future studies. Processing was aimed to finish within a maximum of $3 \mathrm{~h}$ after venipuncture. The samples were centrifuged and thereafter stored at $-80{ }^{\circ} \mathrm{C}$ (without antioxidant). To analyze $\mathrm{FA}$ concentrations, EDTA plasma samples were picked and transported to the Division of Metabolic Diseases and Nutritional Medicine, Dr. von Hauner Children's Hospital, University of Munich Medical Center, in 2010. After being thawed, the analysis of plasma glycerophospholipid FA (hereafter referred to as "plasma FA") composition was performed by a sensitive and precise high-throughput method described recently (24). The average coefficient of variation was $15.7 \%$.

Plasma samples had previously undergone one thaw-freeze cycle. In order to explore the impact of this extra thaw-freeze cycle, 105 new plasma samples from the same series of aliquot tubes were randomly selected for reanalysis, and intraclass correlation coefficients for all 25 FA concentrations were calculated using a two-way mixed-effects model for single measures with absolute agreement. Substantial to almost perfect agreement between the two assessments was found (intraclass correlation coefficients: 0.61-0.96; intraclass correlation coefficient for sum of FAs: 0.87 ), with only one exception for C22:5n-3 (intraclass correlation coefficient: 0.59 ).

In the current study, we used AA, EPA, and DHA. The n-3:n- 6 ratio was calculated by summing the available n-3 PUFAs and dividing this by the sum of the corresponding n- 6 PUFAs in the pathway (see

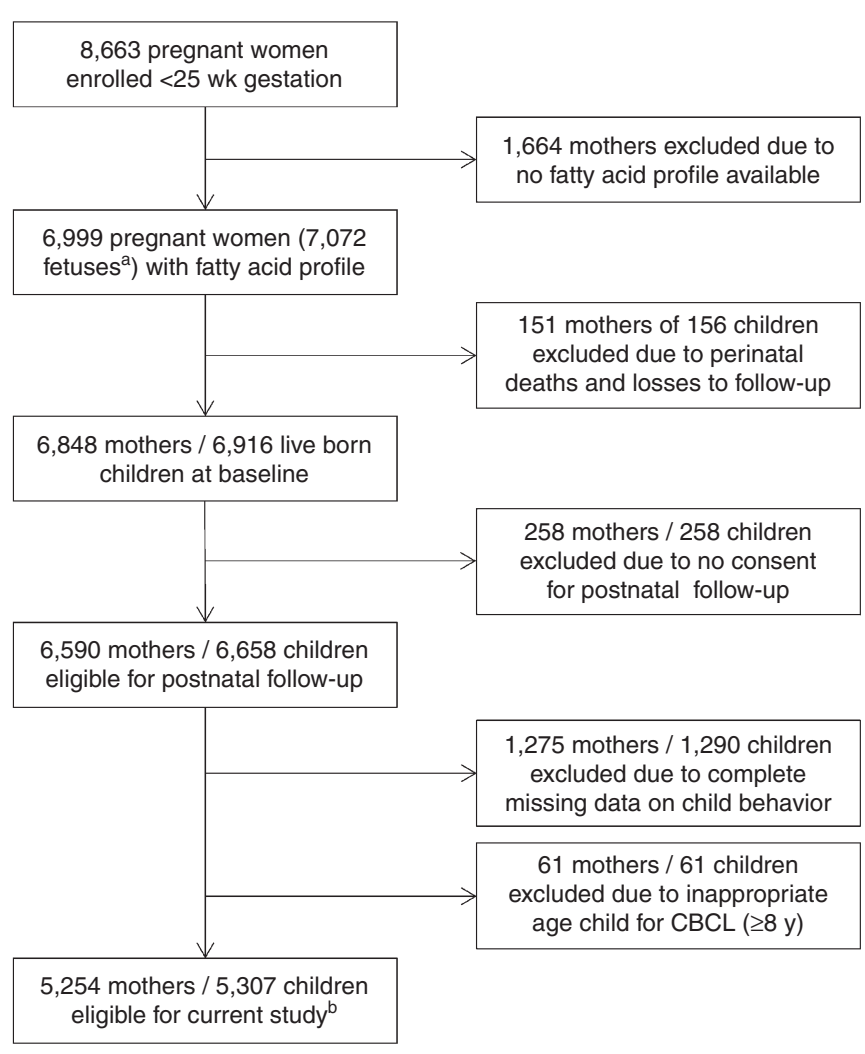

Figure 1. Flow chart of study population. $\mathrm{CBCL}$, child behavior checklist; aDue to twin pregnancies. ${ }^{b}$ Analytic sample, missing imputed by multiple imputation.
Supplementary Table S1 online for an overview). FAs are expressed as percentage by weight (wt\%) of all glycerophospholipid FAs detected with a chain length between 14 and 22 carbon atoms.

\section{Child Emotional and Behavioral Problems}

Mothers were asked to fill out the CBCL when their child was about 6 $y$ old. Since the majority of the children were younger than $6(58.7 \%)$, we used the CBCL $1 \frac{1 / 2}{-5}$ for all children. Additionally, children 8 y or older were excluded from the analyses $(n=61)$. In our sample, for all scales, Cronbach's alphas were the same in 5-y-old children and in children older than 5 , indicating that problems were also reliably measured in children older than 5 .

The CBCL is a self-administered parents-report questionnaire to measure the degree of children's problem behavior (25). It contains 99 problem items rated on a 3 -point scale $(0=$ not true, $1=$ somewhat or sometimes true, and 2 = very true or often true), based on the preceding 2 mo. A broad-band emotional problems (i.e., being anxious, withdrawn, and emotionally reactive) and behavioral problems (i.e., inattention and aggression) scale can be computed. Clinical cutoff scores were used to classify children as having emotional or behavioral problems (91st percentile of a Dutch norm group (26)).

The TRF 6-18 is the teacher version of the CBCL 6-18. Good reliability and validity have been reported for the CBCL and TRF $(25,27)$. In total, TRF data was available in 3,366 children whose mother had a fatty acid profile. Clinical cutoff scores (91st percentile of the middle norm group from multicultural samples $(27,28)$ were used to classify children as having emotional or behavioral problems.

Correlations between parent and teacher report were modest $\left(r_{\text {CBCL- }}\right.$ $=0.19$ for emotional problems; $r_{\mathrm{CBCL}-\mathrm{TRF}}=0.22$ for behavioral problems), which is in line with literature on cross-informant agreement (13) and reflects important variability in behavior across different contexts.

Parent and teacher scores were also combined by averaging the individual age-standardized sum scores. Since no clinical cutoffs were available from norm populations, we used the 91st percentile in our study sample as a cutoff to define child emotional and behavioral problems in the clinical range.

DSM-IV-based diagnosis of problem behavior was obtained by the DISC-YC in a subsample of the cohort when children were about 6 y old. The DISC-YC is a highly structured DSM-IV-based parent-administered interview that generates DSM-IV diagnoses in four modules: anxiety disorders, mood disorders (emotional), behavioral disorders, and miscellaneous (elimination and tic disorders). Good overall reliability of symptom scales has been published (29). DISC-YC administration in the total Generation R cohort was aimed at children enriched for problem behavior (i.e., scoring above the 85th percentile on CBCL total problems or above the 98th percentile of CBCL syndrome scales; $n=1,080$ ) plus a random comparison group $(n=330)$, resulting in oversampling of children at risk for diagnosis. In the current study, interview data were available for 839 children whose mother had a FA profile. Of these, 98 children were classified with a diagnosis "emotional problems with mild impairment" and 221 with "behavioral problems with mild impairment." For each of the cases, four matched (on sex and European origin) controls were selected from the sample with CBCL data available (children scoring below the 50th percentile on both CBCL emotional and behavioral problems). Controls were selected from a total eligible control group of 2,134 children, comprising of 117 children who were not diagnosed with emotional or behavioral problems based on the DISC-YC and an additional group of 2,017 children without interview data.

\section{Covariates}

Several maternal and child characteristics were considered as possible confounding variables, based on previous studies of perinatal LC-PUFA status and child emotional and behavioral development (9-12). These were: family income, maternal educational level, prepregnancy BMI, age at enrollment, smoking and alcohol consumption during pregnancy, national origin, general psychiatric symptoms in mid-pregnancy (highest prevalence for anxious and depressive symptoms), parity, and marital status. Child characteristics that were considered comprised gestational age and weight at birth, sex, 
breastfeeding status at 6 mo, daycare attendance during early childhood, and age of the child at the time of assessment.

All analyses of maternal LC-PUFA status and child problem behavior were controlled for gestational age at venipuncture, sex of the child, and the age of the child at assessment, as well as the other FAs (to test the independent effect of each LC-PUFA). Other covariates were included in the adjusted models if they changed the effect estimates meaningfully (defined as more than 5\%). Following this criterion, maternal pre-pregnancy BMI and child gestational age and weight at birth and breastfeeding status at 6 mo were excluded from the analyses.

We did not have information on child LC-PUFA levels. Instead, we used dietary n-3 and n-6 intake during infancy, as assessed by a 211item Food Frequency Questionnaire when children were 14 mo old (30). N-3 and n-6 intake were adjusted for total energy intake using the residual method (31) and were available for 2,785 of the children in the current study sample.

\section{Statistical Analyses}

To minimize bias resulting from rater perspectives, we used the parent (CBCL, DISC-YC), teacher (TRF), and combined parent/teacher scores of child emotional and behavioral problems as outcome variables.

First, multivariable logistic regression analyses were used to test for associations of maternal AA, EPA, and DHA levels (wt\%) as well as the maternal n-3:n-6 ratio (per SD) with child emotional and behavioral problems using the CBCL, TRF, and the combined CBCL/TRF scores. For the DISC-YC data, we used a case-control approach with conditional logistic regression analysis.

We added quadratic terms of the LC-PUFA variables to test for nonlinear associations. Analyses were repeated while excluding preterm children $(<37 \mathrm{wk}$ of gestation) and children with low birth weight $(<2,500 \mathrm{~g})(n=396)$ and adjusting for child dietary $\mathrm{n}-3$ and $\mathrm{n}-6$ intake at the age of 14 mo in a subsample with these data available.

Next, we explored potential effect modification by child sex and maternal educational level in the fully adjusted models using parent reports. If the interaction term between maternal n-3:n-6 ratio and the effect modifier was statistically significant $(P<0.05)$, we performed additional stratified analyses.

Finally, for ease of interpretation, we divided the n-3:n-6 ratio by quintiles in analyses using parent report of child problem behavior.

Missing values on covariates and child outcome data (when at least one other CBCL measure at 1.5 or $3 \mathrm{y}$ was available) were imputed using the Markov Chain Monte Carlo multiple imputation technique with Predictive Mean Matching for continuous variables (0.1-19.9\% missing data) and generating five datasets. Statistical analyses were carried out using PASW Statistics, version 20.0 for Windows (SPSS, Chicago, IL).

For the nonresponse analysis, we compared child and maternal characteristics of included participants $(n=5,307 ; 76.7 \%)$ with those from whom we did not obtain behavioral data $(n=1,609)$. Excluded children on average had a $108 \mathrm{~g}(95 \% \mathrm{CI}: 76,140 ; P<0.001)$ lower birth weight compared with included children. The mothers of children not included on average had a $0.2 \mathrm{wt} \%(95 \% \mathrm{CI}: 0.17,0.29 ; P<0.001)$ lower DHA level in mid-pregnancy, were on average 3.3 y $(95 \% \mathrm{CI}$ : $3.0,3.6 ; P<0.001)$ younger, and less educated $(22.5 \%$ primary education compared with $8.1 \% ; \chi^{2}=520$ (2); $\left.P<0.001\right)$. They also had more severe psychopathological complaints (median: 0.25 (95\% range: $0-1.98)$ compared with 0.15 (95\% range: $0-1.29) ; P<0.001$ ).

\section{SUPPLEMENTARY MATERIAL}

Supplementary material is linked to the online version of the paper at http://www.nature.com/pr

\section{ACKNOWLEDGMENTS}

The Generation R Study is conducted by the Erasmus Medical Center in close collaboration with the School of Law and the Faculty of Social Sciences at the Erasmus University, Rotterdam; the Municipal Health Service, Rotterdam area; and the Stichting Trombosedienst \& Artsenlaboratorium Rijnmond (Star-MDC), Rotterdam. We gratefully acknowledge the contribution of participating mothers, general practitioners, hospitals, midwives, and pharmacies in Rotterdam, The Netherlands.

\section{STATEMENT OF FINANCIAL SUPPORT}

The general design of the Generation R Study was made possible by financial support from the Erasmus Medical Center, Rotterdam; the Erasmus University Rotterdam; the Dutch Ministry of Health, Welfare and Sport; and The Netherlands Organization for Health Research and Development (ZonMw). The present study was additionally supported by a grant from ZonMw (The Hague, The Netherlands) grant no: 10.000.1003) and a research grant from the European Community's 7th Framework Programme (FP7/2008-2013) under grant agreement 212652 (NUTRIMENTHE project, "The Effect of Diet on the Mental Performance of Children"). The work of H.T. is supported by NWO-ZonMW VIDI grant no: 017.106 .370 . The work of B.K. is supported by European Research Council Advanced grant ERC-2012-AdG- no: 322605 META-GROWTH.

Disclosure: F.C.V. is a contributing author of the Achenbach System of Empirically Based Assessment, from which he receives remuneration. The other authors did not declare any conflict of interest.

\section{REFERENCES}

1. Lauritzen L, Hansen HS, Jørgensen MH, Michaelsen KF. The essentiality of long chain n-3 fatty acids in relation to development and function of the brain and retina. Prog Lipid Res 2001;40:1-94.

2. Yehuda S. Omega-6/omega-3 ratio and brain-related functions. In: Simopoulos AP, Cleland LG, eds. Omega-6/Omega-3 Essential Fatty Acid Ratio: The Scientific Evidence. World Rev Nutr Diet. Basel: Karger, 2003:37-56.

3. Uauy R, Mena P, Rojas C. Essential fatty acids in early life: structural and functional role. Proc Nutr Soc 2000;59:3-15.

4. Gil-Sánchez A, Demmelmair H, Parrilla JJ, Koletzko B, Larqué E. Mechanisms involved in the selective transfer of long chain polyunsaturated Fatty acids to the fetus. Front Genet 2011;2:57.

5. Hibbeln JR, Davis JM, Steer C, et al. Maternal seafood consumption in pregnancy and neurodevelopmental outcomes in childhood (ALSPAC study): an observational cohort study. Lancet 2007;369:578-85.

6. Gale CR, Robinson SM, Godfrey KM, Law CM, Schlotz W, O'Callaghan FJ. Oily fish intake during pregnancy-association with lower hyperactivity but not with higher full-scale IQ in offspring. J Child Psychol Psychiatry 2008;49:1061-8.

7. Waylen A, Ford T, Goodman R, Samara M, Wolke D. Can early intake of dietary omega-3 predict childhood externalizing behaviour? Acta Paediatr 2009;98:1805-8.

8. Chen HF, Su HM. Exposure to a maternal n-3 fatty acid-deficient diet during brain development provokes excessive hypothalamic-pituitary-adrenal axis responses to stress and behavioral indices of depression and anxiety in male rat offspring later in life. J Nutr Biochem 2013;24:70-80.

9. Dunstan JA, Simmer K, Dixon G, Prescott SL. Cognitive assessment of children at age 2(1/2) years after maternal fish oil supplementation in pregnancy: a randomised controlled trial. Arch Dis Child Fetal Neonatal Ed 2008;93:F45-50.

10. Krabbendam L, Bakker E, Hornstra G, van Os J. Relationship between DHA status at birth and child problem behaviour at 7 years of age. Prostaglandins Leukot Essent Fatty Acids 2007;76:29-34.

11. Kohlboeck G, Glaser C, Tiesler C, et al.; LISAplus Study Group. Effect of fatty acid status in cord blood serum on children's behavioral difficulties at $10 \mathrm{y}$ of age: results from the LISAplus Study. Am J Clin Nutr 2011;94: $1592-9$.

12. Loomans EM, Van den Bergh BR, Schelling M, Vrijkotte TG, van Eijsden M. Maternal long-chain polyunsaturated fatty acid status during early pregnancy and children's risk of problem behavior at age 5-6 years. J Pediatr 2014;164:762-8.

13. Rescorla LA, Bochicchio L, Achenbach TM, et al. Parent-teacher agreement on children's problems in 21 societies. J Clin Child Adolesc Psychol 2014;43:627-42.

14. Innis SM, de La Presa Owens S. Dietary fatty acid composition in pregnancy alters neurite membrane fatty acids and dopamine in newborn rat brain. J Nutr 2001;131:118-22.

15. Kodas E, Galineau L, Bodard S, et al. Serotoninergic neurotransmission is affected by $\mathrm{n}-3$ polyunsaturated fatty acids in the rat. J Neurochem 2004;89:695-702. 
16. Romijn HJ, Hofman MA, Gramsbergen A. At what age is the developing cerebral cortex of the rat comparable to that of the full-term newborn human baby? Early Hum Dev 1991;26:61-7.

17. Al MD, van Houwelingen AC, Kester AD, Hasaart TH, de Jong AE, Hornstra G. Maternal essential fatty acid patterns during normal pregnancy and their relationship to the neonatal essential fatty acid status. Br J Nutr 1995; 74:55-68.

18. Georgieff MK, Innis SM. Controversial nutrients that potentially affect preterm neurodevelopment: essential fatty acids and iron. Pediatr Res 2005;57(5 Pt 2):99R-103R.

19. Kawakita E, Hashimoto M, Shido O. Docosahexaenoic acid promotes neurogenesis in vitro and in vivo. Neuroscience 2006;139:991-7.

20. Coti Bertrand P, O'Kusky JR, Innis SM. Maternal dietary (n-3) fatty acid deficiency alters neurogenesis in the embryonic rat brain. J Nutr 2006;136:1570-5.

21. Olivier JD, Akerud H, Kaihola H, et al. The effects of maternal depression and maternal selective serotonin reuptake inhibitor exposure on offspring. Front Cell Neurosci 2013;7:73.

22. Assisi A, Banzi R, Buonocore C, et al. Fish oil and mental health: the role of $\mathrm{n}-3$ long-chain polyunsaturated fatty acids in cognitive development and neurological disorders. Int Clin Psychopharmacol 2006;21:319-36.

23. Jaddoe VW, van Duijn CM, Franco OH, et al. The Generation R Study: design and cohort update 2012. Eur J Epidemiol 2012;27:739-56.
24. Glaser C, Demmelmair H, Koletzko B. High-throughput analysis of fatty acid composition of plasma glycerophospholipids. J Lipid Res 2010;51:216-21.

25. Achenbach TM, Rescorla LA. Manual for the ASEBA Preschool Forms \& Profiles. Burlington, VT: University of Vermont, Research Center for Children, Youth, and Families, 2000.

26. Tick NT, van der Ende J, Koot HM, Verhulst FC. 14-year changes in emotional and behavioral problems of very young Dutch children. J Am Acad Child Adolesc Psychiatry 2007;46:1333-40.

27. Achenbach TM, Rescorla LA. Manual for the ASEBA School-Age Forms \& Profiles. Burlington, VT: University of Vermont, Research Center for Children, Youth, and Families, 2001.

28. Achenbach TM, Rescorla LA. Multicultural Supplement to the Manual for the ASEBA School-Age Forms \& Profiles. Burlington, VT: University of Vermont, Research Center for Children, Youth, and Families, 2007.

29. Lavigne JV, Hopkins J, Gouze KR, et al. Is smoking during pregnancy a risk factor for psychopathology in young children? A methodological caveat and report on preschoolers. J Pediatr Psychol 2011;36:10-24.

30. Kiefte-de Jong JC, de Vries JH, Bleeker SE, et al. Socio-demographic and lifestyle determinants of 'Western-like' and 'Health conscious' dietary patterns in toddlers. Br J Nutr 2013;109:137-47.

31. Willett WC, Howe GR, Kushi LH. Adjustment for total energy intake in epidemiologic studies. Am J Clin Nutr 1997;65:Suppl 4:1220S-8S; discussion 1229S-1231S. 